\title{
Performance evaluation of routing with load-balancing in multi-radio wireless mesh networks
}

\begin{abstract}
Wireless mesh networks (WMNs) face several challenges such as architectural design and network protocol design issues. The capacity of WMNs is very limited, such as the result in an available single channel bandwidth system compared to a multi-channel system. Two problems affect the capacity of mesh networks; i.e. load balancing and interference. To support the mesh network infrastructure, it is necessary to balance the traffic load and reduce the interference. One important direction for improving the capacity of WMNs is to use multiple radio interfaces and multiple channels simultaneously. The proposed load balancing routing algorithm method provides the load balance for multi-radio mesh networks by using a good routing metric, which captures the differences in transmission rates, packet loss ratio, traffic load and intra/inter flow interferences. The simulation results of this study, which used the network simulation NS-2, showed the capacity improvement that helps distributing the traffic load for efficient resource utilization.
\end{abstract}

Keyword: Wireless mesh network; Routing metric; Multi-radio; Multi-channel; Load balancing 